\title{
Immunohistochemical staining patterns of p53 can serve as a surrogate marker for TP53 mutations in ovarian carcinoma: an immunohistochemical and nucleotide sequencing analysis
}

\author{
Anna Yemelyanova ${ }^{1}$, Russell Vang ${ }^{1,2}$, Malti Kshirsagar ${ }^{1,5}$, Dan Lu ${ }^{1}$, Morgan A Marks ${ }^{4}$, \\ Ie Ming Shih ${ }^{1,2,3}$ and Robert J Kurman ${ }^{1,2,3}$ \\ ${ }^{1}$ Department of Pathology, Johns Hopkins University, School of Medicine, Baltimore, MD, USA; ${ }^{2}$ Department \\ of Gynecology and Obstetrics, Johns Hopkins University, School of Medicine, Baltimore, MD, USA; \\ ${ }^{3}$ Department of Oncology, Johns Hopkins University, School of Medicine, Baltimore, MD, USA and \\ ${ }^{4}$ Department of Epidemiology, Johns Hopkins University, School of Public Health, Baltimore, MD, USA
}

\begin{abstract}
Immunohistochemical staining for p53 is used as a surrogate for mutational analysis in the diagnostic workup of carcinomas of multiple sites including ovarian cancers. Strong and diffuse immunoexpression of p53 is generally interpreted as likely indicating a TP53 gene mutation. The immunoprofile that correlates with wild-type TP53, however, is not as clear. In particular, the significance of completely negative immunostaining is controversial. The aim of this study was to clarify the relationship of the immunohistochemical expression of p53 with the mutational status of the TP53 gene in ovarian cancer. A total of 57 ovarian carcinomas ( 43 high-grade serous ovarian/peritoneal carcinomas, 2 malignant mesodermal mixed tumors (carcinosarcomas), 2 low-grade serous carcinomas, 4 clear cell carcinomas, 1 well-differentiated endometrioid carcinoma, and 5 carcinomas with mixed epithelial differentiation) were analyzed for TP53 mutations by nucleotide sequencing (exons 4-9), and subjected to immunohistochemical analysis of p53 expression. Thirty six tumors contained functional mutations and 13 had wild type TP53. Five tumors were found to harbor known TP53 polymorphism and changes in the intron region were detected in three. Tumors with wild-type TP53 displayed a wide range of immunolabeling patterns, with the most common pattern showing $\leq 10 \%$ of positive cells in 6 cases $(46 \%)$. Mutant TP53 was associated with $60-100 \%$ positive cells in 23 cases (64\% of cases). This pattern of staining was also seen in three cases with wildtype TP53. Tumors that were completely negative ( $0 \%$ cells staining) had a mutation of $T P 53$ in $65 \%$ of cases and wild-type TP53 in 11\%. Combining two immunohistochemical labeling patterns associated with TP53 mutations ( $0 \%$ and $60-100 \%$ positive cells), correctly identified a mutation in $94 \%$ of cases $(P<0.001)$. Immunohistochemical analysis can be used as a robust method for inferring the presence of a TP53 mutation in ovarian carcinomas. In addition to a strong and diffuse pattern of p53 expression (in greater than $60 \%$ of cells), complete absence of p53 immunoexpression is commonly associated with a TP53 mutation. Accordingly, this latter pattern, unlike low-level expression (10-50\% cells), should not be construed as indicative of wild-type TP53.

Modern Pathology (2011) 24, 1248-1253; doi:10.1038/modpathol.2011.85; published online 6 May 2011
\end{abstract}

Keywords: immunohistochemistry; mutation; nucleotide sequencing; ovarian cancer; TP53

Correspondence: Dr A Yemelyanova, MD, Division of Gynecologic Pathology, Department of Pathology, The Johns Hopkins Hospital, Weinberg Building, Room 2242, 401 N. Broadway, Baltimore, MD 21231, USA.

E-mail: ayemely1@jhmi.edu

${ }^{5}$ Current address: Clinical Pathology Associates, Austin, TX, USA Received 10 March 2011; revised 5 April 2011; accepted 6 April 2011; published online 6 May 2011
Mutations of the TP53 gene are the most common and most frequently studied molecular alterations in human cancer. ${ }^{1-3}$ Many studies have investigated their significance in diagnosis, prognosis, and treatment in tumors of various sites. For example, the presence of a TP53 mutation in colorectal, lung, prostate, and breast carcinomas has been shown to be a poor prognostic factor, and for the tumors of 
other sites a TP53 mutation has been associated with chemoresistance in some studies. ${ }^{4-9}$ Accordingly, detection of a TP53 mutation has taken on considerable importance in clinical practice.

While nucleotide sequencing is the most reliable technique to detect gene mutation, it is labor intensive, time consuming, and therefore, currently has limited application in clinical pathology practice. Immunohistochemical analysis of p53 expression is therefore commonly used as a surrogate for mutational analysis. ${ }^{10-13}$ It has been generally accepted that wild-type p53 protein is relatively unstable and has a short half-life, which makes it undetectable by immunohistochemistry. ${ }^{14}$ In contrast, mutant p53 has a much longer half-life, and therefore, accumulates in the nucleus creating a stable target for immunohistochemical detection. $^{15,16}$ Although some studies have described immunohistochemical overexpression of wild-type p53, suggesting abnormal stability of non-mutant protein due to impaired degradation under cellular stress, strong and diffuse immunolabeling is generally regarded as indicative of a missense mutation of TP53. ${ }^{1,7,17}$ It has been proposed, but not as well appreciated that complete lack of immunohistochemical expression may be a result of a nonsense mutation leading to formation of a truncated, nonimmunoreactive protein. ${ }^{18-20}$ In view of these different patterns of immunoexpression associated with mutant TP53, it is therefore not surprising that studies correlating TP53 gene status and/or p53 overexpression with clinical outcome and response to chemotherapy in ovarian/pelvic serous carcinoma have reported conflicting results. ${ }^{10,12,19,21-27}$ Some of the studies that focused on ovarian high-grade serous carcinomas failed to demonstrate correlation between TP53 mutation and survival that to some extent have been related to the fact that TP53 mutation is an early event in high-grade serous carcinogenesis with near ubiquitous occurrence. ${ }^{21,25,28,29}$ In addition, accurate pathological classification of ovarian carcinoma is frequently lacking in many reports as evidenced by the use of the all encompassing diagnosis 'ovarian cancer', without segregating tumors by histological type. Particularly pertinent to the current study has been a failure to use a consistent definition of what constitutes p53 overexpression. Some studies have simply used a cuff-off of as little as $10 \%$ of positive cells staining, whereas other have employed complicated scoring systems, which take into account both the quantity of positive cells, as well as the staining intensity. ${ }^{22,25,27,30,31}$ In addition to the lack of uniform scoring systems, variations in immunohistochemical protocols make data from different studies difficult to compare and interpret. The aim of this study was to correlate immunohistochemical staining patterns of p53 expression in ovarian carcinomas with mutational analysis in order to establish practical immunohistochemical cut-points, which can be used to infer the presence of a TP53 mutation.

\section{Materials and methods}

\section{Case Selection and Mutational Analysis}

A total of 57 cases of ovarian carcinoma, which included 43 high-grade serous ovarian/peritoneal carcinomas, 2 malignant mesodermal mixed tumors (MMMTs), 2 low-grade serous carcinomas, 4 clear cell carcinomas, 1 well-differentiated endometrioid carcinoma, and 5 carcinomas with mixed epithelial differentiation (4 mixed serous-endometrioid and 1 serous-clear cell-endometrioid carcinomas) were studied. Tumors that were previously analyzed for TP53 mutation by nucleotide sequencing (exons 4-9) were subjected to immunohistochemical analysis of p53 expression. The details of the mutational analysis have been previously reported. ${ }^{28,29}$ Tumors with no identified mutations in exons 4-9 were considered to have wild-type TP53.

\section{Immunohistochemistry}

One tumor-rich section per case was selected for immunohistochemical analysis. Immunoperoxidase labeling was performed with the automated XT iVIEW DAB V.1 procedure on the BenchMark XT IHC/ISH Staining Module, Ventana with anti-p53 (clone Bp53-11, prediluted, Ventana). Antigen retrieval was carried out with CC1 (Ventana). Sections were incubated with primary antibodies for $16 \mathrm{~min}$ at $37^{\circ} \mathrm{C}$. Staining was detected with I-View DAB detection system. All slides were reviewed by two pathologists (AY and MK). Cases with discordant results underwent a consensus review at a multiheaded microscope with a third pathologist (RV). Nuclear staining was considered a positive reaction. The extent of staining was estimated to the nearest $10 \%$ level of positive tumor cells. The intensity of staining was recorded as weak, moderate, or strong.

\section{Statistical Analysis}

Statistical analysis was carried out using Fisher's exact test (STATA 11.0, STATACORP, College Station, Texas, CA, USA). $P$-values less than 0.05 were considered statistically significant.

\section{Results}

Of the 57 tumors, 36 contained functional mutations and 13 had wild-type TP53. Because of the limited number of cases with known p53 polymorphism $(n=5)$ and changes in the intron region $(n=3)$, these were not included in further analysis. Only functional mutations (ie, changes in nucleotide sequence that led to structural changes of the encoded p53 protein) were considered for statistical analysis.

Of 43 high-grade serous carcinomas, 30 (70\%) tumors contained functional mutations (nucleotide deletion -7 , nucleotide insertion -2 , nucleotide 
substitution -18, and nonsense mutation in 3), known polymorphisms, and changes in the intron region were found in seven tumors; no mutations were identified in six tumors.

Five tumors with mixed epithelial differentiation (4 mixed serous-endometrioid and 1 serous-clear cell-endometrioid carcinomas) all contained functional mutations (nucleotide deletion -one, nucleotide substitution -four). All five tumors displayed greater than $60 \%$ positive cells pattern of immunostaining (90\% positive cells pattern was seen in four carcinomas and $60 \%$ positive cells pattern in one).

In two MMMTs, one had nucleotide deletion with complete absence of immunoexpression; the other contained known polymorphism with $80 \%$ positive cells immunostaining pattern.

One low-grade endometrioid adenocarcinoma arising in a background of an atypical proliferative (borderline) endometrioid tumor had wild-type TP53 and less than $10 \%$ positive cells immunostaining pattern.

No mutations were identified in four clear cell carcinomas and two low-grade serous carcinomas, all of which had less than $10 \%$ positive cells.

Immunohistochemical and mutational analysis data are summarized in Table 1. All tumors with $10 \%$ of positive cells demonstrated predominately weak staining with occasional nuclei displaying moderate intensity of staining.

Among carcinomas with wild-type TP53, one of two tumors with $20-50 \%$ of positive cells displayed strong to moderate staining intensity, the other case in this group showed weak staining. All tumors with $60-100 \%$ of positive cells demonstrated strong staining, with a few cases displaying areas of moderate intensity in addition to strongly staining areas.

In 23 cases $(63 \%)$ with mutant $T P 53 \geq 60 \%$ of tumor cells were positive for p53 (Figure 1). Nineteen of these cases contained missense mutations. Additionally, 11 cases (31\%) with mutations were completely negative for p53 (Figure 2). This immunolabeling pattern was associated with nucleotide deletion and nonsense mutations in 9 of 11 cases $(82 \%)$. By combining two immunohistochemical staining patterns (strong and diffuse expression and complete lack of expression), the immunohistochemical analysis correlated with the mutational analysis in $94.4 \%$ of cases $(P<0.001$, Fisher's exact test). Tumors with wild-type TP53 $(n=13)$ displayed a wide range of immunolabeling patterns, with the most common pattern being $\leq 10 \%$ of positive cells present in six cases $(46 \%)$ (Figure 3).

Table 1 Immunohistochemical staining patterns and mutational analysis of p53 in ovarian carcinomas

\begin{tabular}{|c|c|c|c|c|c|c|c|}
\hline \multirow[b]{2}{*}{$\begin{array}{l}\text { Immunohistochemical } \\
\text { staining pattern } \\
\text { ( } \% \text { positive cells) }\end{array}$} & \multicolumn{7}{|c|}{ Mutational analysis } \\
\hline & $\begin{array}{l}\text { Wild } \\
\text { type }\end{array}$ & $\begin{array}{l}\text { Nucleotide } \\
\text { deletion }\end{array}$ & $\begin{array}{l}\text { Nucleotide } \\
\text { insertion }\end{array}$ & $\begin{array}{l}\text { Missense } \\
\text { mutation }\end{array}$ & $\begin{array}{l}\text { Nonsense } \\
\text { mutation }\end{array}$ & $\begin{array}{c}\text { Known TP53 } \\
\text { polymorphism }\end{array}$ & $\begin{array}{l}\text { Changes } \\
\text { in intron } \\
\text { region }\end{array}$ \\
\hline Negative $(n=17)$ & 2 & 7 & 1 & 1 & 2 & 2 & 2 \\
\hline$\leq 10 \%(n=8)$ & 6 & - & - & 2 & - & - & - \\
\hline $90-100 \%(n=19)$ & 1 & 1 & 1 & 14 & 1 & 1 & - \\
\hline Total $(n=57)$ & 13 & 9 & 2 & 22 & 3 & 5 & 3 \\
\hline
\end{tabular}
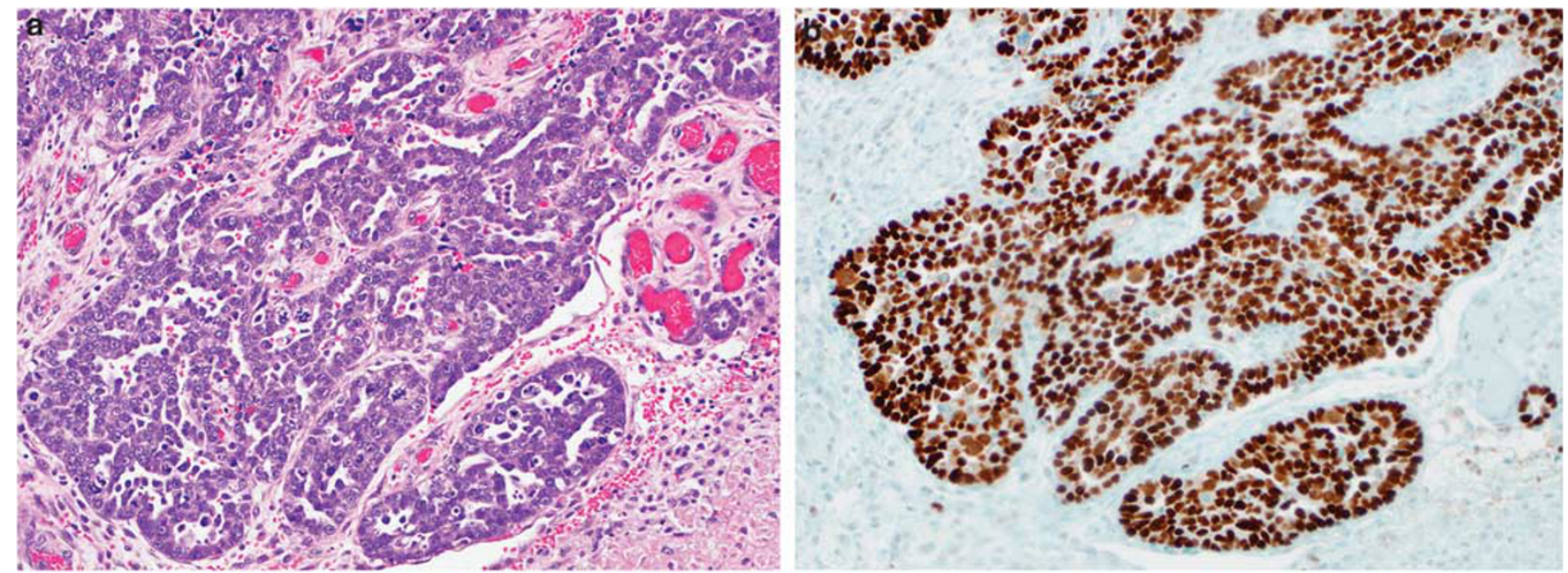

Figure 1 High-grade serous carcinoma (missense mutation, exon 6) (a); strong and diffuse immunoexpression of p53 (100\% of positive cells) (b). 

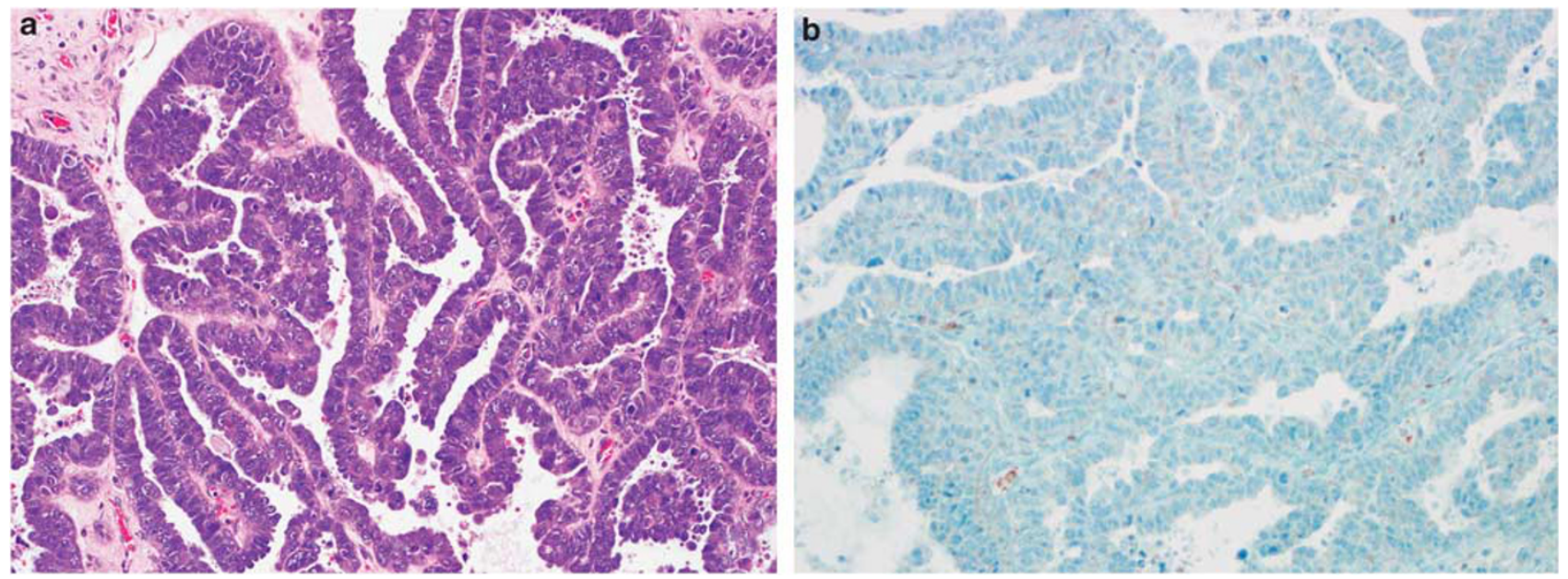

Figure 2 High-grade serous carcinoma (nucleotide insertion, exon 4) (a); completely absent immunoexpression of p53 (0\% of positive cells) with weakly positive stromal cells serving as an internal positive control (b).
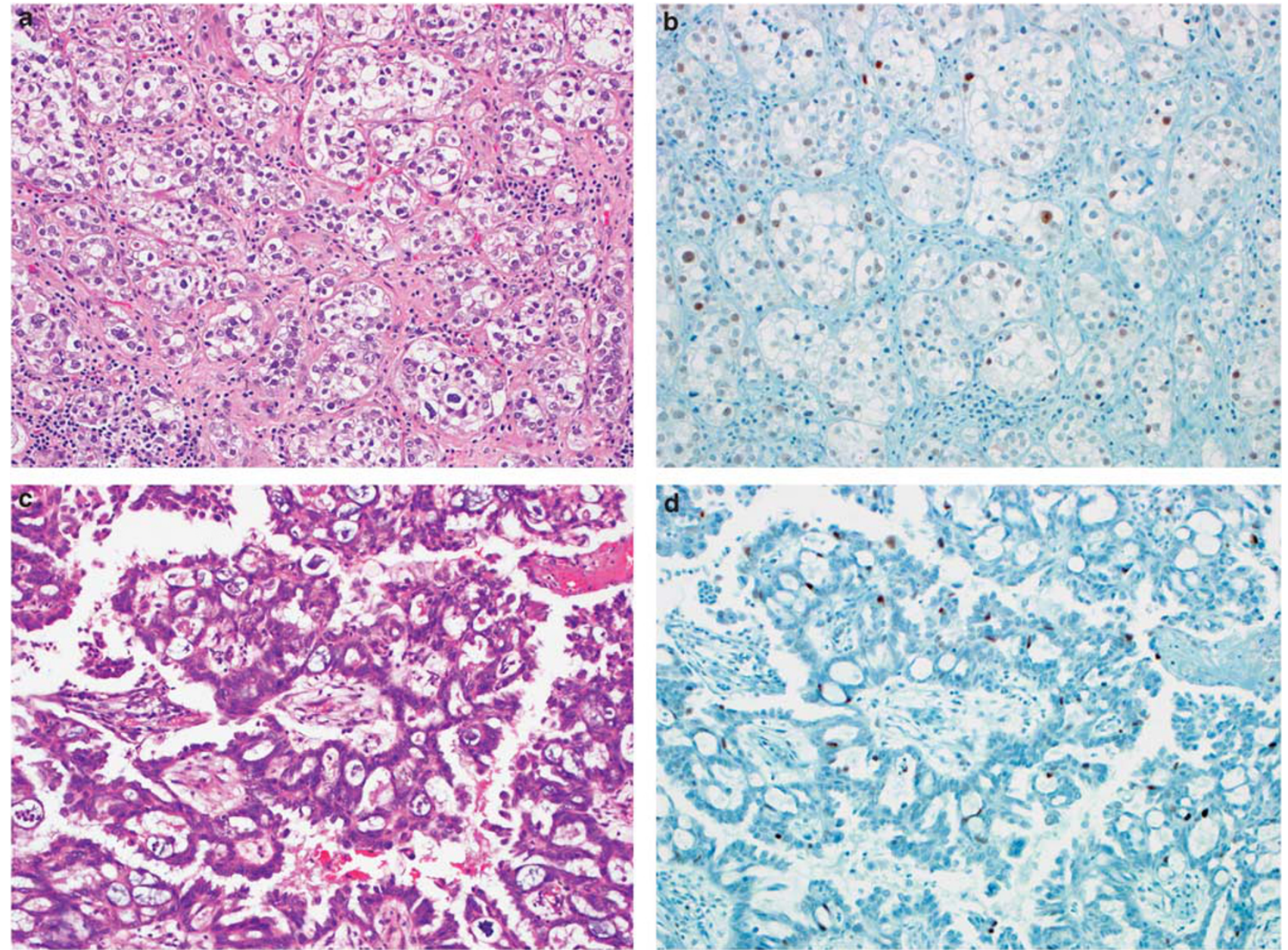

Figure 3 Clear cell carcinoma (a), and low-grade serous carcinoma (c) with wild-type TP53. Rare p53-positive cells ( $<10 \%$ cells positive) $(\mathbf{b}, \mathbf{d})$.

\section{Discussion}

In this study, p53 expression was scored as the percent of positive tumor cells. This simple method was easy to apply compared to complex scoring schemes that take into account combination of staining distribution (percent of positive cells), as well as staining intensity. Parenthetically, all cases 
with 60-100\% positive tumor cells demonstrated strong-to-moderate staining intensity, therefore, inclusion of staining intensity into immunohistochemical scoring scheme would not improve performance of the test at the chosen cut-off. In addition, evaluating intensity of staining is problematic as it is difficult to reproduce and it can vary with different protocols.

Known TP53 polymorphisms do not represent gene mutation and most of them are expected to be phenotypically silent. ${ }^{32,33}$ Moreover, it is not entirely clear whether TP53 polymorphisms may or may not result in conformational changes leading to altered immunoreactivity of p53. ${ }^{34}$ Because of these issues and due to the small number of such cases in this study, we chose not to analyze them. We also excluded tumors with changes in the intron region, as it was not possible to determine whether these changes could have caused a frameshift and resulted in a protein with aberrant immunoexpression.

The findings in this study confirm that immunohistochemical overexpression of p53 ( $\geq 60 \%$ positive cells) closely correlates with a TP53 mutation. Even more importantly, it was found that complete absence of immunolabeling was also indicative of a mutation. Our results therefore support previously published data reporting that missense mutations in the TP53 gene lead to formation of a stable protein resulting in immunohistochemical overexpression, whereas nucleotide deletions and non-sense mutations result in protein truncation and complete lack of immunolabeling. ${ }^{18,20}$

The lack of p53 immunoexpression associated with nonsense (or null) mutation is very important to recognize because some studies demonstrated worse prognosis for patients with this finding compared with patients whose tumor had a missense mutation. ${ }^{19,35}$ If less than $50 \%$ positive tumor cells (not including cases completely lacking immunoexpression) are used as a cut-off level, it would correctly identify $61.5 \%$ of cases with wildtype TP53. More importantly, if two patterns of immunolabeling commonly associated with TP53 mutation (60-100\% of tumor cells positive and tumors completely negative for p53) were combined, immunohistochemical analysis would correlate with mutations in almost $95 \%$ of cases.

Accordingly, p53 immunohistochemical scoring systems should not interpret complete absence of expression as consistent with wild-type TP53. It should also be emphasized that evaluation of cases with complete lack of immunoexpression should be carried out with strict attention to external and internal controls in order to ensure that the negative result is not due to a technically suboptimal immunohistochemical preparation. The presence of rare weakly positive nuclei (a pattern commonly associated with wild type TP53) in stroma or adjacent non-neoplastic tissue present in the same section can serve as a useful internal positive control. Evaluation of the complete absence of immunoexpression should be made exclusively in tumor cells, as lack of immunoexpression can be observed in normal non-neoplastic epithelium where it does not indicate TP53 mutation.

There are several possible explanations for the lack of perfect correlation between TP53 mutation and immunohistochemical expression. First, the mutational analysis in this study evaluated codons 4 through 9, which would identify most, but not all possible mutated loci. ${ }^{21,36}$ Second, it has been suggested that cellular stress may result in delayed degradation of wild-type p53 making it detectable by immunohistochemistry. ${ }^{37}$ Third, since the sample on which mutational analysis was carried out was not the same as the sample that was immunostained, it is conceivable that tumor heterogeneity could account for the discordant findings in tumors acquiring TP53 mutation as a late event in carcinogenesis. In conclusion, immunohistochemical staining for p53 can be used as a surrogate for mutational analysis in ovarian carcinomas, if tumors that are diffusely positive ( $\geq 60 \%$ of cells) and those that are completely negative are interpreted as indicative of a mutation. Additional studies of tumors from other sites are necessary in order to determine whether the findings in ovarian carcinoma can be generalized to other tumor types.

\section{Disclosure/conflict of interest}

The authors declare no conflict of interest.

\section{References}

1 Bennett WP, Hollstein MC, Hsu IC, et al Mutational spectra and immunohistochemical analyses of p53 in human cancers. Chest 1992;101:19S-20S.

2 Nigro JM, Baker SJ, Preisinger AC, et al Mutations in the p53 gene occur in diverse human tumour types. Nature 1989;342:705-708.

3 Hollstein M, Sidransky D, Vogelstein B, et al p53 mutations in human cancers. Science 1991;253:49-53.

4 Bunz F, Hwang PM, Torrance C, et al Disruption of p53 in human cancer cells alters the responses to therapeutic agents. J Clin Invest 1999;104:263-269.

5 Chang H, Jiang AM, Qi CX. Aberrant nuclear p53 expression predicts hemizygous 17p (TP53) deletion in chronic lymphocytic leukemia. Am J Clin Pathol 2010;133:70-74.

6 Hussain SP, Hofseth LJ, Harris CC. Tumor suppressor genes: at the crossroads of molecular carcinogenesis, molecular epidemiology and human risk assessment. Lung Cancer 2001;34(Suppl 2):S7-S15.

7 Iggo R, Gatter K, Bartek J, et al Increased expression of mutant forms of p53 oncogene in primary lung cancer. Lancet 1990;335:675-679.

8 Russo A, Bazan V, Iacopetta B, et al The TP53 colorectal cancer international collaborative study on the prognostic and predictive significance of p53 mutation: influence of tumor site, type of mutation, 
and adjuvant treatment. J Clin Oncol 2005;23: 7518-7528.

9 Smith ND, Rubenstein JN, Eggener SE, et al The p53 tumor suppressor gene and nuclear protein: basic science review and relevance in the management of bladder cancer. J Urol 2003;169:1219-1228.

10 Hall PA, Lane DP. p53 in tumour pathology: can we trust immunohistochemistry? Revisited! J Pathol 1994; 172:1-4.

11 Lepelley P, Preudhomme C, Vanrumbeke M, et al Detection of p53 mutations in hematological malignancies: comparison between immunocytochemistry and DNA analysis. Leukemia 1994;8:1342-1349.

12 Marks JR, Davidoff AM, Kerns BJ, et al Overexpression and mutation of p53 in epithelial ovarian cancer. Cancer Res 1991;51:2979-2984.

13 Wynford-Thomas D. P53 in tumour pathology: can we trust immunocytochemistry? J Pathol 1992;166:329-330.

14 Rogel A, Popliker M, Webb CG, et al p53 cellular tumor antigen: analysis of mRNA levels in normal adult tissues, embryos, and tumors. Mol Cell Biol 1985;5: 2851-2855.

15 Finlay CA, Hinds PW, Tan TH, et al Activating mutations for transformation by p53 produce a gene product that forms an hsc70-p53 complex with an altered half-life. Mol Cell Biol 1988;8:531-539.

16 Kraiss S, Spiess S, Reihsaus E, et al Correlation of metabolic stability and altered quaternary structure of oncoprotein p53 with cell transformation. Exp Cell Res 1991;192:157-164.

17 Bartek J, Iggo R, Gannon J, et al Genetic and immunochemical analysis of mutant p53 in human breast cancer cell lines. Oncogene 1990;5:893-899.

18 Lax SF, Kendall B, Tashiro $\mathrm{H}$, et al The frequency of p53, K-ras mutations, and microsatellite instability differs in uterine endometrioid and serous carcinoma: evidence of distinct molecular genetic pathways. Cancer 2000;88:814-824.

19 Shahin MS, Hughes JH, Sood AK, et al The prognostic significance of p53 tumor suppressor gene alterations in ovarian carcinoma. Cancer 2000;89:2006-2017.

20 Tashiro $\mathrm{H}$, Isacson $\mathrm{C}$, Levine $\mathrm{R}$, et al p53 gene mutations are common in uterine serous carcinoma and occur early in their pathogenesis. Am J Pathol 1997;150:177-185.

21 Ahmed AA, Etemadmoghadam D, Temple J, et al Driver mutations in TP53 are ubiquitous in high grade serous carcinoma of the ovary. J Pathol 2010;221:49-56.

22 Darcy KM, Brady WE, McBroom JW, et al Associations between p53 overexpression and multiple measures of clinical outcome in high-risk, early stage or suboptimally-resected, advanced stage epithelial ovarian cancers A Gynecologic Oncology Group study. Gynecol Oncol 2008;111:487-495.

23 Kerns BJ, Jordan PA, Moore MB, et al p53 overexpression in formalin-fixed, paraffin-embedded tissue detected by immunohistochemistry. J Histochem Cytochem 1992;40:1047-1051.
24 Kmet LM, Cook LS, Magliocco AM. A review of p53 expression and mutation in human benign, low malignant potential, and invasive epithelial ovarian tumors. Cancer 2003;97:389-404.

25 Laframboise S, Chapman W, McLaughlin J, et al p53 mutations in epithelial ovarian cancers: possible role in predicting chemoresistance. Cancer J 2000;6: 302-308.

26 Singer G, Stohr R, Cope L, et al Patterns of p53 mutations separate ovarian serous borderline tumors and low- and high-grade carcinomas and provide support for a new model of ovarian carcinogenesis: a mutational analysis with immunohistochemical correlation. Am J Surg Pathol 2005;29:218-224.

27 Wen WH, Reles A, Runnebaum IB, et al p53 mutations and expression in ovarian cancers: correlation with overall survival. Int J Gynecol Pathol 1999;18:29-41.

28 Salani R, Kurman RJ, Giuntoli R, et al Assessment of TP53 mutation using purified tissue samples of ovarian serous carcinomas reveals a higher mutation rate than previously reported and does not correlate with drug resistance. Int J Gynecol Cancer 2008;18: 487-491.

29 Vang R, Shih I, Salani R, et al Subdividing ovarian and peritoneal serous carcinoma into moderately differentiated and poorly differentiated does not have biologic validity based on molecular genetic and in vitro drug resistance data. Am J Surg Pathol 2008;32:1667-1674.

30 Hartmann LC, Podratz KC, Keeney GL, et al Prognostic significance of p53 immunostaining in epithelial ovarian cancer. J Clin Oncol 1994;12:64-69.

31 Havrilesky L, Darcy M, Hamdan H, et al Prognostic significance of p53 mutation and p53 overexpression in advanced epithelial ovarian cancer: a Gynecologic Oncology Group Study. J Clin Oncol 2003;21: 3814-3825.

32 Moreau F, Matlashewski G. Molecular analysis of different allelic variants of wild-type human p53. Biochem Cell Biol 1992;70:1014-1019.

33 Beckman G, Birgander R, Sjalander A, et al Is p53 polymorphism maintained by natural selection? Hum Hered 1994;44:266-270.

34 Thomas M, Kalita A, Labrecque S, et al Two polymorphic variants of wild-type p53 differ biochemically and biologically. Mol Cell Biol 1999;19:1092-1100.

35 Hashimoto T, Tokuchi Y, Hayashi M, et al p53 null mutations undetected by immunohistochemical staining predict a poor outcome with early-stage non-small cell lung carcinomas. Cancer Res 1999;59:5572-5577.

36 Casey G, Lopez ME, Ramos JC, et al DNA sequence analysis of exons 2 through 11 and immunohistochemical staining are required to detect all known p53 alterations in human malignancies. Oncogene 1996;13:1971-1981.

37 Zaika A, Marchenko N, Moll UM. Cytoplasmically 'sequestered' wild type p53 protein is resistant to Mdm2-mediated degradation. J Biol Chem 1999;274: 27474-27480. 